\title{
CAPITULO 38
}

\section{INTERVENÇÕES NÃO FARMACOLÓGICAS PARA O \\ ENFRENTAMENTO DA PANDEMIA DA COVID -19: \\ RELATO DE EXPERIÊNCIA}

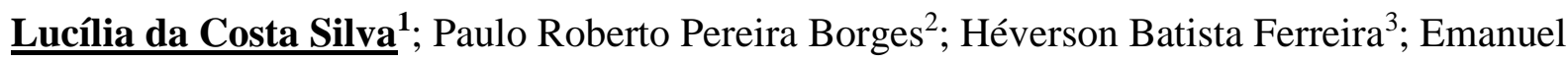
Osvaldo de Sousa ${ }^{4}$; Maria da Conceição Lopes de Oliveira ${ }^{5}$; Diego Bruno Brito Cerqueira ${ }^{6}$; Francisca Taysa de Abreu Silva ${ }^{7}$; Daniel da Silva Gomes ${ }^{8}$; João Felipe Tinto Silva ${ }^{9}$;Karoline Carvalho da Silva Amaro ${ }^{10}$

${ }^{1}$ Centro Universitário Santo Agostinho-UNIFSA, (luciliafisio@outlook.com)

${ }^{2}$ Universidade Estadual do Piauí- UESPI, (ppereiraborges@gmail.com)

${ }^{3}$ Universidade: Universidade Potiguar - UNP, (eversonbatst@gmail.com)

${ }^{4}$ Centro Universitário UNIFACID, (emanfisio@ hotmail.com)

${ }^{5}$ Faculdade do Piauí - FAPI, (ceicinha_lopes@hotmail.com)

${ }^{6}$ Centro Universitário UNIFACID, (diegocerqueirafisioterapeuta@ hotmail.com)

${ }^{7}$ Centro Universitário Santo Agostinho-UNIFSA, (taysa.abreu163@gmail.com)

${ }^{8}$ Centro Universitário Santo Agostinho - UNIFSA, (danielsgoms@gmail.com)

${ }^{9}$ Centro Universitário de Ciências e Tecnologia do Maranhão, (felipetinto99@gmail.com)

${ }^{10}$ Faculdade Adventista da Bahia(FADBA), (karolinecarvalhosilva @ gmail.com)

\section{Resumo}

Objetivo: Relatar sobre as intervenções não farmacológicas e prevenir o novo coronavírus (COVID-19) no Município de Cabeceiras do Piauí. Método: De forma articulada, baseada nas melhores evidências atuais, de modo a se favorecer a adesão das pessoas às intervenções não farmacológicas(INF), uma equipe multidisciplinar realizou intervenções com orientações a adesão das pessoas as INF em três barreiras sanitárias. Resultados: Foram 248 participantes, a princípio eram convidados para um diálogo sobre educação em saúde, com o foco nas INF para enfrentamento da pandemia no seu dia a dia. Considerações Finais: Intervenções em atenção primária, como a proporcionada pela equipe multiprofissional no combate ao COVID -19 é importante para reduzir a transmissão desse vírus, pois a prevenção é um dos métodos que vem apresentando efetividade na diminuição da evolução do pico de agravos.

\section{E - book Pesquisa e Tecnologia: Protagonismo e} Inovações 
Palavras-chave: Coronavírus; COVID-19; Pandemia.

Área Temática: Selecionar uma das áreas temáticas do Congresso.

E-mail do autor principal: luciliafisio@outlook.com

\section{INTRODUÇÃO}

O novo vírus SARS-CoV2 agente etiológico do coronavírus (COVID-19), espalhou-se rapidamente, pelo mundo, logo após sua primeira ocorrência em Wuhan, na China, em dezembro de 2019(WONG et al, 2020). E com o crescimento no número de casos, óbitos e países afetados, a Organização Mundial da Saúde (OMS) declarou que o evento constituía uma Emergência de Saúde Pública de Importância Internacional (ESPII) em 30 de janeiro de 2020. Em 03 de fevereiro, o Ministério da Saúde através da portaria $N^{\circ} 188 / 2020$, afirmou Emergência em Saúde Pública de Importância Nacional (ESPIN). O primeiro caso de Covid19 no Brasil foi confirmado em 26 de fevereiro de 2020, logo após a OMS declarou a pandemia de COVID-19, em 11 de março (GARCIA et al.,2020).

No Brasil, e em diversos países do mundo, a resposta sanitária tem sido centrada nos serviços hospitalares, com ações para a ampliação do número de leitos, especialmente, de unidades de tratamento intensivo e respiradores pulmonares. Sem retirar a importância da adequada estruturação da atenção especializada voltada aos casos mais graves da COVID-19, é preciso alertar que, no âmbito da atenção primária à saúde (APS), muito pode e precisa ser feito. Entretanto é possível reduzir a demanda instantânea por cuidados de saúde e mitigar as consequências da doença sobre a saúde das populações, incluindo a minimização da morbidade e da mortalidade associadas (GARCIA et al, 2020; MEDINA et al 2020).

O Estado do Piauí, apresentou os primeiros casos confirmados no início do mês de março, na capital Teresinense e posteriormente em vários munícipios. No qual, profissionais da Saúde do Município de Cabeceiras do Piauí, em 19 de maio de 2020, notificou seu primeiro caso, atualmente se estendendo a diversos casos confirmados e óbitos. Segundo Qualls et al., (2020), intervenções não Farmacológicas (INF) para o enfrentamento da pandemia da COVID -19 são medidas de saúde pública com alcance individual, ambiental e comunitário. As medidas individuais incluem a lavagem das mãos, a etiqueta respiratória e o distanciamento social. $\mathrm{O}$ distanciamento social, por sua vez, abrange o isolamento de casos, a quarentena aplicada a contatos, e a prática voluntária de não frequentar locais com aglomerações de pessoas

No enfrentamento à pandemia, as práticas de educação em saúde devem envolver minimamente três segmentos: (1) profissionais de saúde comprometidos com o SUS, com a

\section{E - book Pesquisa e Tecnologia: Protagonismo e Inovações}


defesa da vida e que valorizem ações de prevenção da doença, promoção de saúde e práticas de cuidado; (2) gestores que apoiem e implementem políticas de educação em saúde; e (3) população, na busca de autonomia nos cuidados individuais e coletivos (CECCON et al., 2020).

A partir dessa premissa, a Figura 1, apresenta as principais medidas de saúde pública que foram adotadas de forma precoce visando reduzir o impacto da pandemia, já na figura 2 demonstra a curva hipotética mostrando o curso normal da epidemia e o achatamento da curva esperado com a adoção de intervenções não farmacológicas.

\section{Distanciamento Social}

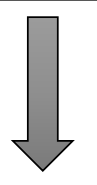

Imediato isolamento de infectados e suspeitos. Orientação para ás medidas de prevenção.
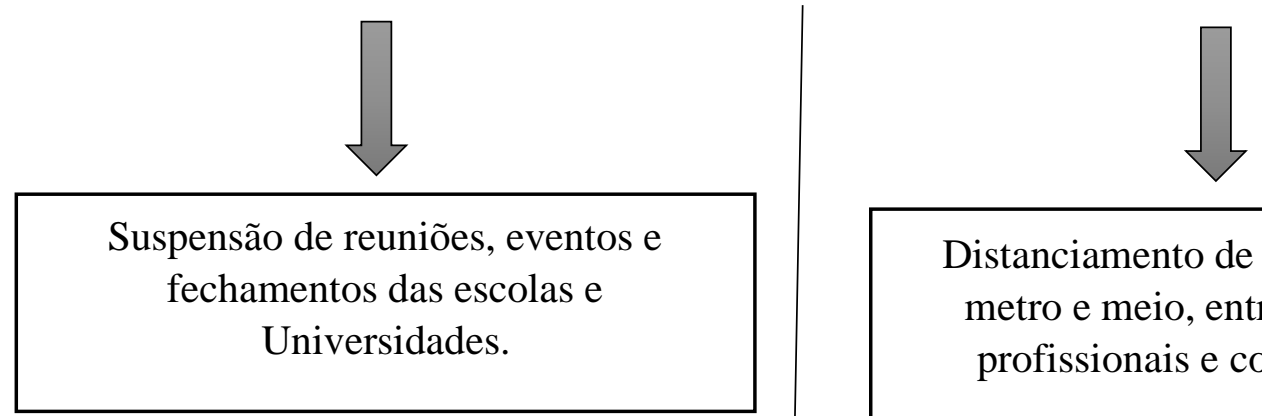

Suspensão de reuniões, eventos e Universidades.
Vigilância Sanitária: Pesquisar

a Intensidade de Transmissão do Sars-CoV-2 para a

comunidade.

Figura 1 - Fluxograma das medidas de contenção da circulação do novo coronavírus, visando a redução do impacto da pandemia por COVID-19. (EUROPEAN CENTRE FOR DISEASE PREVENTION AND CONTROL, 2020). 


\section{ISBN 978-65-995353-4-5}

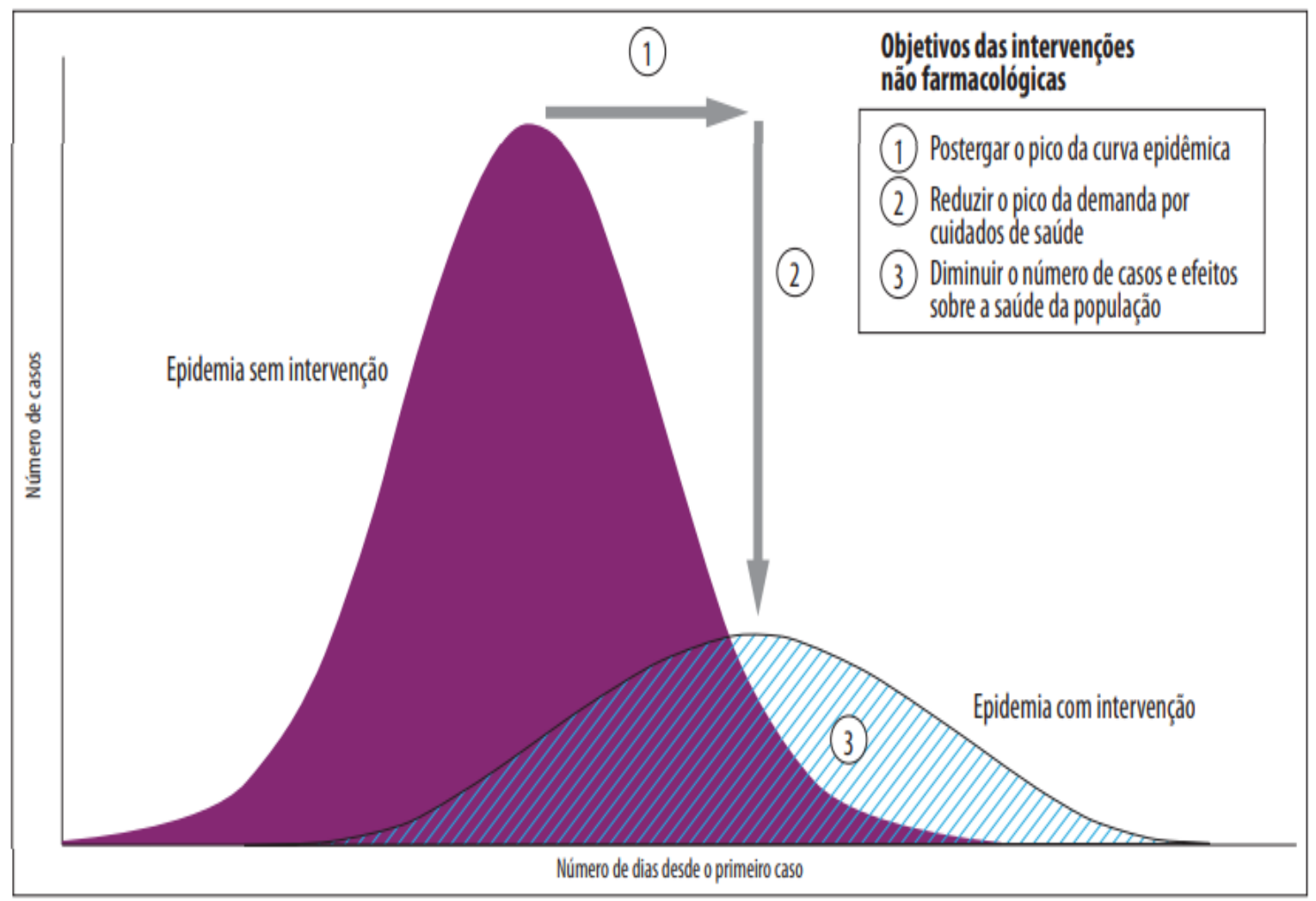

Figura 02

Fonte: Adaptado do Centro e Prevenção de Doenças, SOURCE et al., 2007; QUALLS et.al., 2017.

Diante disso, o presente estudo teve como objetivo primordial, orientar sobre uso das intervenções não farmacológicas e prevenir a transmissibilidade do novo Coronavírus em Cabeceiras do Piauí.

\section{MÉTODO}

Essa pesquisa trata-se de um relato de experiência, sobre as intervenções em três Barreiras Sanitárias, que ocorreu nos dias (07 à 12 de Abril de 2020). A princípio, era realizado um convite para um diálogo sobre educação em saúde, sendo três equipes subdivididas, nas principais divisas que dão acesso ao Município de Cabeceiras do Piauí. De forma articulada, baseada nas melhores evidências atuais, de modo a se favorecer a adesão das pessoas às INF, uma equipe multidisciplinar realizou intervenções com 248 participantes (de diversos gêneros e idades). As equipes de profissionais da saúde e polícia militar, faziam uso de equipamentos de proteção individual (EPI) bem como: máscaras, luvas, tocas de cabelos, álcool gel 70\%, avental, crachás de identificação profissional, fichas padronizada e folhetos informativos (figuras 3 e 4 ). Inovações 


\section{ISBN 978-65-995353-4-5}

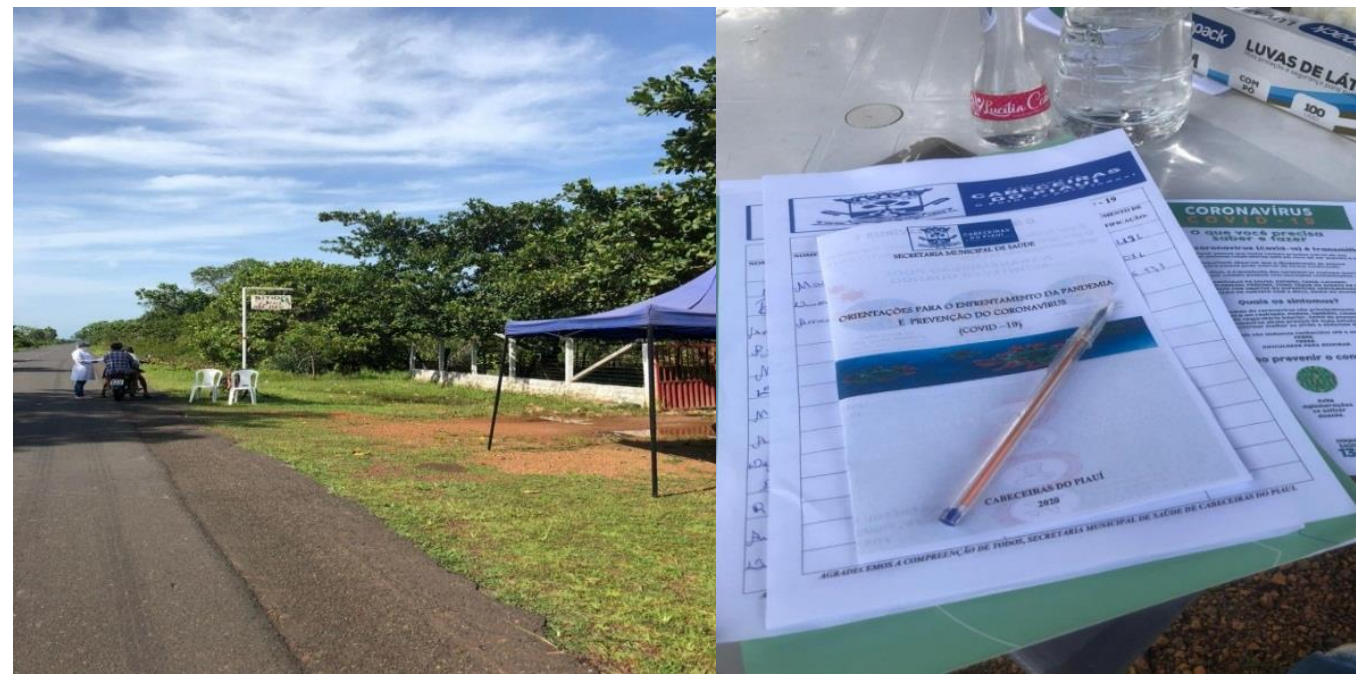

Fonte: Os autores, 2021.

\section{RESULTADOS E DISCUSSÃO}

Ao consolidar todos os dados, dos dias (07 à 12 de Abril de 2020), obtivemos 224 participantes, que relatavam portar seus documentos pessoais de identificação, Cartão Nacional do Sistema Único de Saúde (SUS) e tivemos uma média de 24 indivíduos, que não andavam com o cartão do (SUS), documentos esses necessários e de grande importância ao trafegarem para outra(s) cidades, não somente em casos de urgência e emergência. No total 248 indivíduos, receberam orientações sobre a efetividade da INF para a prevenção do COVID-19.

Gráfico 1: Apresenta os dados em porcentagem, referente a quantidade de participantes, que trafegavam em uma das divisas. Sendo 134 (Barras-Cabeceiras, 84 José de Freitas e 30 Campo Maior).

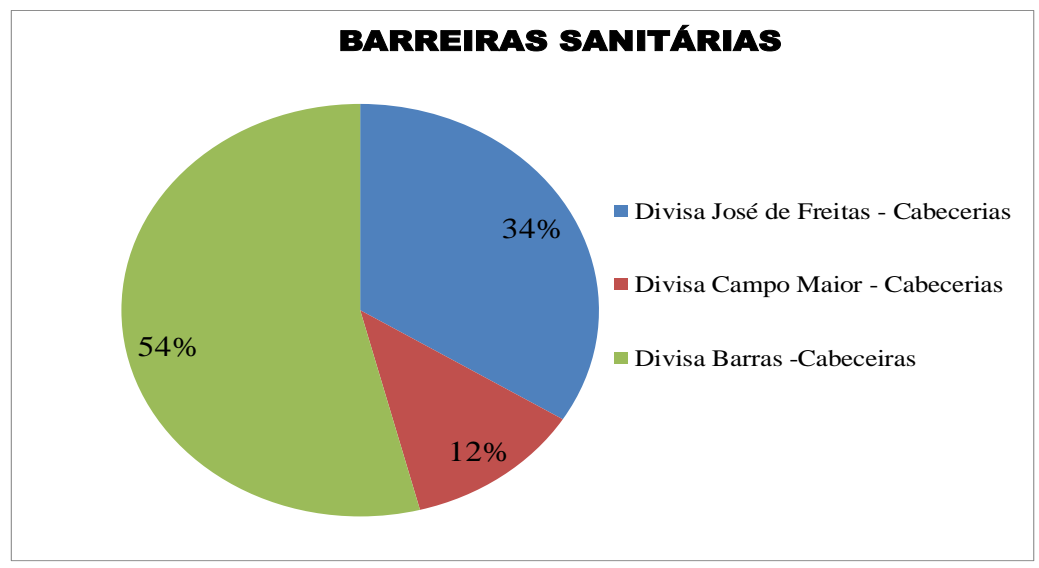

Fonte: Autores,2021.

Ao longo das ações em atenção primária, observamos que os idosos, apresentam uma maior resistência quanto ao distanciamento social e prevenção, alguns relataram ter um ou mais Inovações 
diagnóstico de doenças crônicas. E isso é preocupante, pois os idosos costumam ser mais vulneráveis a doenças infectocontagiosas e a COVID-19, não é exceção.

Embora o risco de morte pela COVID-19, seja mais elevado em pacientes idosos e/ou naqueles com doenças preexistentes, o relato dos primeiros casos nos Estados Unidos, publicado pelo Centro de Controle de Doenças (CDC), constatou que 38\% dos hospitalizados têm idade de 20 a 54 anos (CENTER FOR DISEASE CONTROL, 2020). Portanto, a doença não se limita aos chamados grupos de risco, podendo acometer a população em sua totalidade (WORLD HEALTH ORGANIZATION, 2020a). Corroborando, nesse contexto, são indicadas as INF, visando inibir a transmissão entre humanos, desacelerar o espalhamento da doença, e consequentemente diminuir e postergar o pico de ocorrência na curva epidêmica. Contudo o isolamento social, pode ser incentivado por todos os profissionais da equipe, principalmente pelos agentes comunitários de saúde (ACS), mobilizando lideranças e recursos locais com ampla divulgação de informações e realização de medidas concretas (ANDERSON et al., 2020; MEDINA et al., 2020).

Assim, o foco são as estratégias de promoção de saúde, prevenção da doença, sinais e sintomas e orientação quanto à rede de atenção à saúde, centrada na prática do isolamento social. É fundamental também a produção pedagógica centrada na prática do isolamento social, buscando encontrar, na singularidade de cada sujeito, os caminhos para superar as dificuldades oriundas do enclausuramento. $\mathrm{O}$ fato de não seguir o isolamento social pode estar relacionado à crise econômica para alguns, enquanto para outros estar relacionado ao tédio e à falta de liberdade, por exemplo. Ambos necessitam práticas educacionais distintas, encontrando as linhas de fuga específicas para superar estas barreiras (CECCON et al., 2020).

A sociedade ela nasce, cresce, deseja, sonha, adoece, ela está viva e atualmente ela para, pedi um pouco mais de paciência, novas adaptações de higiene, proteção, mudanças de hábitos e rotinas, a luta é coletiva, porém todos precisam fazer a sua parte! A OMS, recomenda que precisamos educar plenamente o público em geral sobre a seriedade do COVID-19 e do seu papel na prevenção da propagação da doença (WORLD HEALTH ORGANIZATION, 2020).

No estudo de Source et al., 2020, ele destaca que a adoção de tais medidas tem impactos importantes nas atividades diárias, nas vidas das pessoas e na sociedade. A adoção bemsucedida de restrição social como medida de Saúde Pública(SP) traz comprovados benefícios à redução da taxa de transmissão da COVID-19; entretanto, efeitos negativos, associados a essa restrição, poderão ter consequências para a saúde, no médio e longo prazo. Portanto espera-se movaçoes 
das ações de SP, também, uma capacidade de minimizar os efeitos adversos da restrição social prolongada (MALTA et al., 2020).

\section{CONSIDERAÇÕES FINAIS}

Conclui-se que intervenções em atenção primária, como a proporcionada pela equipe multidisciplinar no combate ao COVID -19, é um dos métodos importante para reduzir a transmissão desse vírus, pois as INF promovem diminuição da evolução do pico de transmissibilidade e agravos. Diante disso, que seja aprofundado e implantadas estratégias para futuras pesquisa, para maior efetividade de evidências científicas referente a pandemia do novo coronavírus, pois a uma escassez na literatura referente a patologia.

\section{REFERÊNCIAS}

ANDERSON, R. M.; HEESTERBEEK, H.; KLINKENBERG, D. et al. How will countrybased mitigation measures influence the course of the COVID-19 epidemic?. The Lancet, v. 395, n. 10228, p. 931-934, 2020.

CENTER FOR DISEASE CONTROL. Coronavirus (COVID-19). Disponível em: <https://www.cdc.gov/coronavirus/2019-ncov/index.html>. Acesso em: 28/12/ 2021.

CECCON R.F.;SCHNEIDER, I.J.C.l. Tecnologias leves e educação em saúde não enfrentamento à pandemia da COVID-19, 2020.

European Centre for Disease Prevention and Control. Considerations relating to social distancing measures in response to the COVID-19 epidemic. Stockholm; 2020.

GARCIA, L.P.; DUARTE, E. Intervenções não farmacológicas para o enfrentamento à epidemia da COVID-19 no Brasil., v. 29, n.2, p:01-07,2020.

MALTA, D. C., SZWARCWALD, C. L., BARROS, M. B. D. A et al. A pandemia da COVID19 e as mudanças no estilo de vida dos brasileiros adultos: um estudo transversal, 2020. Epidemiologia e Serviços de Saúde, v. 29, n.1 , p :1-27, 2020.

MEDINA, M. G., GIOVANELLA, L., BOUSQUAT, A. et al. Atenção primária à saúde em tempos de COVID-19: o que fazer?. Cadernos de Saúde Pública, v. 36,n.8, p:01-05,2020.

Ministério da Saúde (BR). Portaria MS/GM n. 188, de 3 de fevereiro de 2020. Declara Emergência em Saúde Pública de importância Nacional (ESPIN) em decorrência da Infecção Humana pelo novo Coronavírus (2019-nCoV), 2020. (Acesso em 27/12/2021).

QUALLS, N., LEVITT, A., KANADE, N., et al. Community mitigation guidelines to prevent pandemic influenza-United States. MMWR Recommendations and Reports, v. 66, n. 1, p. $1,2017$. Inovações 
SOURCE: Adapted from: CDC. Interim pre-pandemic planning guidance: community strategy for pandemic influenza mitigation in the United States-early, targeted, layered use of nonpharmaceutical interventions. Atlanta, GA: US Department of Health and Human Services, CDC; 2007.

WONG J, GOH QY, TAN Z, et al. Preparing for a COVID-19 pandemic: a review of operating room outbreak response measures in a large tertiary hospital in Singapore. Can J Anesth/J Can Anesth. p. 1-14, 2020.

WORLD HEALTH ORGANIZATION. Infection Prevention and Control during Halth Care When Novel Coronavirus (NCOV) Infection Is Suspected. OMS. Disponível em: $<$ https://www.who.int/publications-detail/infection-prevention-andcontrol-during-health-carewhen-novel-coronavirus-(ncov)-infection-is-suspected20200125, 2020. Acesso em 28/12/2021. 